Check for updates

Cite this: RSC Adv., 2018, 8, 2723

Received 1st December 2017 Accepted 4th January 2018

DOI: $10.1039 / c 7 r a 12948 c$

rsc.li/rsc-advances

\section{Extraction of oil from high-moisture tuna liver by subcritical dimethyl ether: feasibility and optimization by the response surface method $\dagger$}

\begin{abstract}
Yizhou Fang, Saiqi Gu, Shulai Liu, Jianyou Zhang, Yuting Ding and Jianhua Liu (DD*
The purpose of this paper is to explore the possibilities of subcritical dimethyl ether extraction (SDME) of oil from tuna liver with high-moisture content. The results showed that the oil and water were successfully coextracted from the liver, and could be easily separated by centrifugation. In addition, the response surface method was employed to optimize the process parameters of SDME, including temperature/pressure, time and stirring speed. It was predicted that a temperature/pressure of $42 / 0.80{ }^{\circ} \mathrm{C} / \mathrm{MPa}$, time of $50 \mathrm{~min}$ and stirring speed of $925 \mathrm{rpm}$ were the optimum within the experimental ranges, with an oil yield of $17.46 \pm$ $0.23 \%$. Furthermore, supercritical carbon dioxide extraction $\left(\mathrm{SC}-\mathrm{CO}_{2}\right)$ was studied comparatively. Only minor differences were observed between the oils extracted by SDME and SC- $\mathrm{CO}_{2}$, which indicated the high-quality of the SDME-oil. With no freeze-drying procedure and the relatively low pressure used in SDME, SDME could be a promising technique for extraction of marine fish liver oil.
\end{abstract}

\section{Introduction}

In China, tuna (Katsuwonus pelamis) is a popular seafood with a yearly output of 4-6 million tons. ${ }^{1}$ However, a large amount of viscera, especially the liver, is discarded. The liver of marine fish is an excellent precursor to marine oil, which contains a considerable amount of n-3 polyunsaturated fatty acids (PUFAs). In particular, eicosapentaenoic acid (EPA, C20:5, n-3) and docosahexaenoic acid (DHA, C22:6, n-3) are reputed to have functions of reducing low density lipoprotein, blood viscosity, and platelet cohesion, and increasing high density lipoprotein. ${ }^{2}$ Furthermore, tuna liver oil is a particularly potent source of vitamin A and vitamin D. Further investigations ${ }^{3}$ have indicated that vitamin A and vitamin D are essential nutrients because of their important roles in vision, bone health, cellular differentiation, embryonic development, reproduction, and the immune system. Thus, the production of tuna liver oil which is rich in n-3 PUFAs and vitamins is a good opportunity for valorizing fish by-products and increasing the competitiveness of the fish industry.

The common extraction procedures used for fish oil production had been studied comprehensively by Rubiorodríguez et al., ${ }^{4}$ including cold extraction, wet reduction, enzymatic extraction, and supercritical carbon dioxide extraction $\left(\mathrm{SC}-\mathrm{CO}_{2}\right)$. The results indicated that $\mathrm{SC}-\mathrm{CO}_{2}$ was the optimal

Department of Food Science and Engineering, Ocean College, Zhejiang University of Technology, Hangzhou 310014, P. R. China. E-mail: jhliu@zjut.edu.cn; Fax: +86 571 88320237; Tel: +8657188320237

$\uparrow$ Electronic supplementary information (ESI) available. See DOI: $10.1039 / \mathrm{c} 7 \mathrm{ra12948c}$ technology for obtaining high quality fish oil comparing to other extraction processes, because it was very useful for reducing fish oil oxidation and the amount of certain impurities. However, the negative aspects of $\mathrm{SC}^{-\mathrm{CO}_{2}}$ were also apparent. The main limitation of $\mathrm{SC}-\mathrm{CO}_{2}$ was the high cost at production scale, not only because of the high pressure used, but also due to the raw material should be freeze-dried in order to reduce its moisture and keep unaltered the nutrient contents of fish oil. ${ }^{5}$ Thus, there is no doubt that it is meaningful to find a replacement for $\mathrm{SC}-\mathrm{CO}_{2}$ in order to reduce the cost of production and maintain the quality of product. For this purpose, subcritical dimethyl ether extraction (SDME), with the following two advantages: reducing the operation pressure and without the freeze-drying pretreatment, was studied.

Rubiorodríguez et al. ${ }^{4}$ optimized the process parameters on the extraction yield of oil from squid livers by $\mathrm{SC}_{-} \mathrm{CO}_{2}$, which indicated that almost all the oil could be extracted at the conditions of $25 \mathrm{MPa}, 40{ }^{\circ} \mathrm{C}$ and $3 \mathrm{~h}$. However, Goto et al. ${ }^{6}$ found that $98.9 \%$ of oil could be extracted from algae by SDME at $0.68 \mathrm{MPa}, 30{ }^{\circ} \mathrm{C}$ and $40 \mathrm{~min}$. Subcritical fluid extraction, also called pressurized low-polarity fluid extraction, was used in our developed technology. As a modified method of the supercritical fluid extraction, subcritical fluid extraction requires lower temperature and pressure, which means the higher security and the lower cost. ${ }^{7}$ Furthermore, the mild temperature and pressure also reduces the degradation of bioactive components resulting in a richer final product. ${ }^{8}$

Dimethyl ether (DE), with the critical temperature and pressure of $126.85{ }^{\circ} \mathrm{C}$ and $5.37 \mathrm{MPa},{ }^{9}$ respectively, was used as solvent in our study. DE has the following characteristics: (a) DE has been approved as a safe extraction solvent in food industry 
by the Food Safety Authority of Europea; ${ }^{9}$ (b) DE will not present in the final products at room temperature due to the low boiling point $\left(-24.8{ }^{\circ} \mathrm{C}\right) ;^{9}$ (c) DE possessing a potential low toxicity according to observations from inhalation studies; ${ }^{8}$ therefore, in our study, DE would be used in a closed process system and recovered, which not only removes the hidden danger of DE, but also results in cost savings. On the other hand, it is important to control the moisture content of raw material before supercritical or subcritical fluid extraction because the water has a negative entrainer effect on the extractability of oil. ${ }^{10}$ However, different to traditional solvents, DE has a good solubility to both water and oil. ${ }^{11}$ Therefore, SDME has been reported on (i) extraction of oil from high-moisture microalgae, ${ }^{6}$ polluted soil, ${ }^{12}$ yeast and soil fungi; ${ }^{\mathbf{1 3}}$ (ii) extraction of water from highmoisture materials, such as sewage sludge and vegetal biomass; ${ }^{\mathbf{1 0 , 1 4}}$ (iii) extraction of bioactive components, including carotenoids from macroalga and catechins from green tea. ${ }^{15,16}$

However, extraction of oil from high-moisture liver by SDME has not been reported. Thus, the feasibility of extraction of oil from the tuna liver without freezing-drying using low-pressure SDME was studied. Besides, the process parameters of SDME were optimized by response surface method, and the properties of the obtained oil were determined and compared with that obtained by $\mathrm{SC}-\mathrm{CO}_{2}$.

\section{Materials and methods}

\subsection{Materials}

The liver of tuna (Katsuwonus pelamis) was used as raw material from a unique batch (related to a certain place, season of fish capture and processing batch) and supplied by Xingye Industrial Group Co., Ltd. (Zhejiang, China). The tuna liver was delivered frozen at $-18{ }^{\circ} \mathrm{C}$. The liver was packed in individual plastic bags under vacuum and kept frozen until the experiments were performed. Technical grade DE (of 99.95\% purity) was purchased from Jingong Special Gas (Hangzhou, China). All other reagents were analytical grade supplied by Fisher Scientific (Shanghai, China). Standards of fatty acid methyl esters were purchased from Sigma-Aldrich (Shanghai, China).

\subsection{Subcritical DE extraction}

2.2.1. Subcritical extraction apparatus. The subcritical extraction apparatus (see Fig. $\mathrm{S} 1 \dagger$ ) used in this study was supplied by Henan Province Subcritical Biological Technology Co., LTD (Henan, China).

There were three characteristics with this apparatus. Firstly, extraction tank was not equipped with a pressure device for regulating pressure to control internal pressure. In other words, the extraction pressure was fixed under specific conditions (the amount of solvent and material, temperature and stirring speed). Secondly, an agitator was equipped in extraction tank, which could agitate the solvent during extraction. Thirdly, the solvent could be recycled and reused by the compressor, and the cycle of solvent was shown in Fig. 1.

2.2.2. Subcritical DE extraction method. The basic scheme of SDME extraction process was depicted in Fig. 1. The tuna liver was minced $5 \mathrm{~min}$ by an electric mixer before SDME. Besides, in order to minimize the effect of unexplained variability due to the slight differences between raw materials and operations, $3 \mathrm{~kg}$ of tuna live (more than $17 \times 150 \mathrm{~g}$, the required amount of response surface experiment) was processed simultaneously, then the minced tuna live was vacuum packed with $150 \pm 1 \mathrm{~g}$ per bag. The packed liver was stored at $-18{ }^{\circ} \mathrm{C}$ and used after fully thawed in the dark.

Firstly, the quantitative tank, extraction tank and vaporizing tank were submitted to vacuum, which could prevent the oxidation of oil and create the pressure difference between solvent tank and quantitative tank. Secondly, the subcritical DE, which was stored in solvent tank (volume, $15 \mathrm{~L}$ ), flowed to quantitative tank (volume, $3 \mathrm{~L}$ ) under the influence of pressure, and the amount of subcritical DE could be controlled according to the gradations on quantitative tank. Thirdly, the minced liver was loaded into the iron covered with nylon cloth, which could prevent the liver flowing together with the extraction product. Fourthly, the subcritical DE flowed down to extraction tank (volume, $5 \mathrm{~L}$ ), and the subcritical DE was heated by hot-water. Fifthly, the subcritical DE and extraction product were transferred to vaporizing tank (volume, $5 \mathrm{~L}$ ) after extraction, and then the subcritical DE was evaporated, recycled and reused. Sixthly, the extraction tank and vaporizing tank were submitted to vacuum again, which assured that most of the volatile DE was eliminated from the final products. Finally, the mixture of water and oil could discharge from vaporizing tank, and the tuna liver oil could easily separate with water by centrifuging under $1000 \mathrm{~g}$ and $5 \mathrm{~min}$.

\subsection{Supercritical $\mathrm{CO}_{2}$ extraction method}

The Spe-ed ${ }^{\text {Tм }}$ Prime $\mathrm{SC}-\mathrm{CO}_{2}$ system was supplied by Applied Separations Inc. (Allentown, American). The extraction method was according to Sahena et al. ${ }^{17}$ with some modifications.

The tuna liver was previously freeze-dried (FreeZone $12 \mathrm{~L}$, Labconco) for $12 \mathrm{~h}$ before SC- $\mathrm{CO}_{2}$. Amounts of around $15 \mathrm{~g}$ of dried tuna liver were used in each experiment. The $\mathrm{SC}-\mathrm{CO}_{2}$ was carried out under $35 \mathrm{MPa}$ and $50{ }^{\circ} \mathrm{C}$ with a continuous $\mathrm{CO}_{2}$ flow of $3 \mathrm{~mL} \mathrm{~min}{ }^{-1}$. The extraction was accomplished after $4 \mathrm{~h}$, isothermally and at constant pressure, with a yield of $17.51 \pm$ $0.11 \%$.

\subsection{Experimental design}

As described in 2.2.1, the extraction pressure was nonadjustable under specific conditions, including the amount of subcritical DE and liver, temperature and stirring speed. In order to control the pressure during extraction, we fixed the amount of solvent and raw material added and analyzed the effects of temperature and stirring speed to pressure. The amount of subcritical DE added was $3 \mathrm{~L}$, which was the maximum of quantitative tank. Besides, the effect of solid-liquid ratio on the yield of liver oil under excessive conditions was analyzed (Fig. S2 $\dagger$ ), which indicated that the solid-liquid ratio $\left(\mathrm{g} \mathrm{mL}^{-1}\right)$ of 20 was optimal. When the amount of solvent and raw material added were fixed, the effects of extraction temperature and stirring speed on the extraction pressure were studied (Fig. S3 $\dagger$ ). During extraction, 


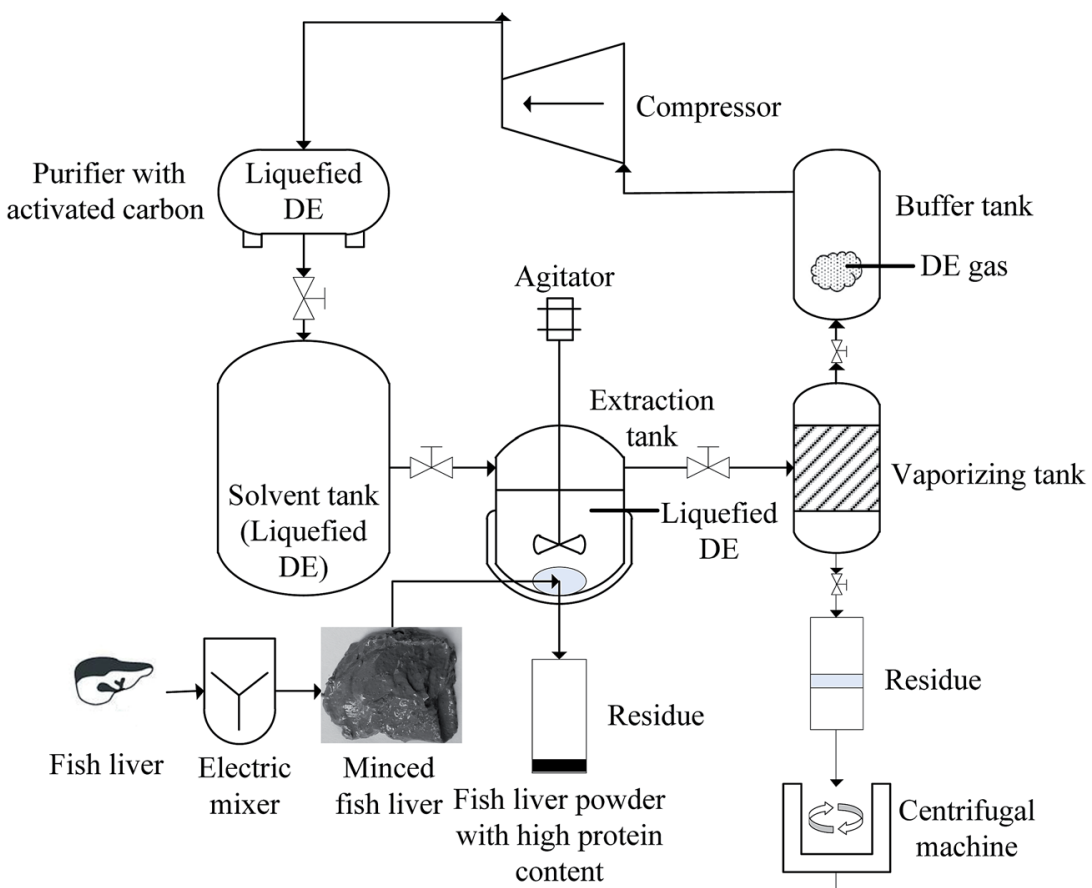

Water

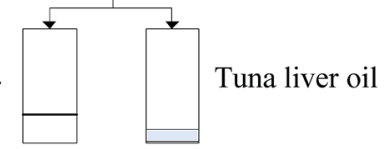

Fig. 1 The basic scheme of the process of extraction of oil from tuna liver.

the extraction tank could be considered as an enclosed system, and the temperature had a positive correlation with pressure, while stirring speed had no significant effect on pressure $(p>$ 0.05). When the temperature varies from $20{ }^{\circ} \mathrm{C}$ to $50{ }^{\circ} \mathrm{C}$, the pressure increased from 0.44 MPa to 0.93 MPa. In summary, the pressure was determined by temperature when the amount of subcritical DE and liver added was unchanged. Thus, temperature and pressure were studied as a factor in our paper, marked temperature/pressure.

Response surface method (software Design-Expert 10.0.1, Stat-Ease, Inc, Minneapolis, U.S.) was applied to optimize the process parameters for SDME as described elsewhere. ${ }^{18,19}$ The temperature/pressure $\left(X_{1}\right)$, time $\left(X_{2}\right)$ and stirring speed $\left(X_{3}\right)$ were studied as independent variables to optimize the yield of tuna liver oil $(Y)$. The $Y$ was calculated as follows:

$$
Y=\frac{\text { Mass of extracted tuna liver oil }}{\text { Mass of tuna liver }}
$$

The preliminary proper ranges of temperature/pressure, extraction time and stirring speed were determined according to the results of single-factor experimentation (Fig. S4†). Thus, we selected $40 / 0.77\left({ }^{\circ} \mathrm{C} / \mathrm{MPa}\right), 45(\mathrm{~min})$ and $750(\mathrm{rpm})$ as the central points for temperature/pressure, extraction time and stirring speed in the response surface method experiments, respectively. Table 1 details the experimental conditions and the results carried out for developing the model. All experiments were carried out in a random order, in order to minimize the effect of unexplained variability of extraneous factors.

\subsection{Analytical methods}

2.5.1. Chemical properties of tuna liver. Experiment analysis adopted the content of moisture ( $\mathrm{Ba} 2 \mathrm{a}-38$ ), fat ( $\mathrm{Ba} 3-38$ ), protein (Ba 4a-38) and ash (Ba 5a-49) as described by $\mathrm{AOCS}^{20}$ to evaluate the changes in composition of tuna liver before and after SDME.

Table 1 Experimental scheme and results obtained from RSM for the oil yield

\begin{tabular}{lllll}
\hline \multicolumn{3}{l}{ Independent variable } & \\
\cline { 2 - 3 } & $\begin{array}{l}X_{1}(\text { temperature } / \\
\left.\text { pressure, }{ }^{\circ} \mathrm{C} / \mathrm{MPa}\right)\end{array}$ & $X_{2}($ time, min) & $\begin{array}{l}X_{3} \text { (stirring } \\
\text { speed, rpm) }\end{array}$ & $\begin{array}{l}\text { Oil yield } \\
(\%)\end{array}$ \\
\hline 1 & $-1(30 / 0.60)$ & $-1(30)$ & $0(750)$ & 14.39 \\
2 & $+1(50 / 0.93)$ & $-1(30)$ & $0(750)$ & 14.91 \\
3 & $-1(30 / 0.60)$ & $+1(60)$ & $0(750)$ & 16.12 \\
4 & $+1(50 / 0.93)$ & $+1(60)$ & $0(750)$ & 16.65 \\
5 & $-1(30 / 0.60)$ & $0(45)$ & $-1(500)$ & 13.34 \\
6 & $+1(50 / 0.93)$ & $0(45)$ & $-1(500)$ & 14.12 \\
7 & $-1(30 / 0.60)$ & $0(45)$ & $+1(1000)$ & 16.78 \\
8 & $+1(50 / 0.93)$ & $0(45)$ & $+1(1000)$ & 17.04 \\
9 & $0(40 / 0.77)$ & $-1(30)$ & $-1(500)$ & 12.25 \\
10 & $0(40 / 0.77)$ & $+1(60)$ & $-1(500)$ & 14.04 \\
11 & $0(40 / 0.77)$ & $-1(30)$ & $+1(1000)$ & 16.07 \\
12 & $0(40 / 0.77)$ & $+1(60)$ & $+1(1000)$ & 17.02 \\
13 & $0(40 / 0.77)$ & $0(45)$ & $0(750)$ & 16.80 \\
14 & $0(40 / 0.77)$ & $0(45)$ & $0(750)$ & 16.90 \\
15 & $0(40 / 0.77)$ & $0(45)$ & $0(750)$ & 16.96 \\
16 & $0(40 / 0.77)$ & $0(45)$ & $0(750)$ & 16.76 \\
17 & $0(40 / 0.77)$ & $0(45)$ & $0(750)$ & 16.84
\end{tabular}


2.5.2. Chemical properties of tuna liver oil. Experiment analysis adopted acid values (Cd 3d-63), peroxide values (Cd 853 ), iodine values (Cd 1-25), non-saponification matter (Ca 6b53), insoluble impurities ( $\mathrm{Ca} \mathrm{3a-46)}$ and moist and volatile matter (Ca 2c-25) as described by $\mathrm{AOCS}^{20}$ to evaluate the quality of tuna liver oil.

2.5.3. The residual of DE. The residual of DE was analyzed by solid phase micro extraction (SPME) coupled with gas chromatography-mass (GC-MS). The SPDE device (Supelco, Beijing, China) was equipped with a needle with a $75 \mu \mathrm{m}$ film with the coating of carboxen/polydimethylsiloxane (CAR/ PDMS). The sample was kept in a water bath for $30 \mathrm{~min}$ at $80{ }^{\circ} \mathrm{C}$, and the volatile compounds from the sample were exposed and adsorbed by SPDE needle. GC-MS was carried out with a Trace 1300 gas chromatograph (Thermo Scientific) equipped a flame ionization detector (FID). The SPDE needle was injected and thermally desorbed at $250{ }^{\circ} \mathrm{C}$ for $3 \mathrm{~min}$. Compounds were separated by a TG-WAXMS column $(30 \mathrm{~m} \times$ $0.25 \mathrm{~mm} \times 0.50 \mu \mathrm{m})$. The column temperature was programmed starting at a constant temperature of $40{ }^{\circ} \mathrm{C}$, further increased to $100{ }^{\circ} \mathrm{C}$ at $4{ }^{\circ} \mathrm{C} \min ^{-1}$ and then increased to $150{ }^{\circ} \mathrm{C}$ at $2{ }^{\circ} \mathrm{C} \min ^{-1}$ and maintained for $2 \mathrm{~min}$.

The SDME tuna live oil samples $(5 \mathrm{~mL})$ were placed in a $15 \mathrm{~mL}$ headspace sampling flask and analyzed. For DE, the headspace sampling flask filled with gas DE was analyzed.

2.5.4. Fatty acids analysis of tuna liver oil. The fatty acids profile was determined by the method of Rubio-Rodríguez et al. ${ }^{5}$ The fatty acid methyl esters were firstly prepared and then analyzed by GC-MS in a Trace 1300 gas chromatograph (Thermo Scientific, USA) equipped with an auto-sampler (AI 1310 series) and a FID. The separation was carried out with helium $\left(1.8 \mathrm{~mL} \mathrm{~min}^{-1}\right)$ as carrier gas. A Supelco SP-2560 column $(100 \mathrm{~m}$ $\times 0.25 \mathrm{~mm} \times 0.20 \mu \mathrm{m})$ was used. The oven temperature was held as follows: $120{ }^{\circ} \mathrm{C}$ for $1 \mathrm{~min}$, increased to $175{ }^{\circ} \mathrm{C}$ at $15{ }^{\circ} \mathrm{C} \min ^{-1}$ and maintained for $10 \mathrm{~min}$, further increased to $210{ }^{\circ} \mathrm{C}$ at $10{ }^{\circ} \mathrm{C} \min ^{-1}$ and maintained for $5 \mathrm{~min}$, and then increased to $260^{\circ} \mathrm{C}$ at $10^{\circ} \mathrm{C} \mathrm{min}^{-1}$ and maintained for $20 \mathrm{~min}$. A split injector $(50: 1)$ at $250{ }^{\circ} \mathrm{C}$ model with a solvent delay of 20 min was used. The FID was heated at $220^{\circ} \mathrm{C}$. Most of the fatty acid methyl esters were identified by comparison of their retention times with those of chromatographic standards.

\subsection{Statistical analysis}

Statistical analysis was performed using IBM SPSS statistics software (v. 20.0, SPSS, Inc., Chicago, IL). The data were expressed as means with standard deviations $(n=3)$, and analyzed by one-way ANOVA and Tukey's test with a 0.05-level of significance.

\section{Results and discussion}

\subsection{The composition of tuna liver}

The contents of moisture, crude protein, total lipids and ash in crude tuna liver were $61.41 \pm 3.12 \%, 16.44 \pm 0.86 \%, 17.60 \pm$ $0.69 \%$ and $0.63 \pm 0.02 \%$, respectively. The values of crude moisture, protein and ash were similar to those measured in literatures, ${ }^{\mathbf{2 1 , 2 2}}$ where the moisture, protein and ash of tuna liver were between $66.7-71.3 \%, 11.48-18.1 \%$ and $1.5-2.37 \%$, respectively, whereas the contents of lipids were lower (2.6$10.1 \%)$. The variations in them may result from genetic, aging factors and the way of extraction.

\subsection{Feasibility analysis}

The pictures of raw material and products at all stages of SDME were shown in Fig. 2.

As expected, water and lipids could be extracted together by SDME. The residue in vaporizing tank was a mixture of water and oil. The oil phase was on top and the water phase was below, with a pretty clear dividing line between them. It was indicated that there was no unexpected reactions occurred between water and oil, such as emulsification. Besides, the tuna liver oil, clear and transparent, could separate and got simply by centrifugation. In addition, the residue in extraction tank was in a form of powder, which also indicated that partial water had been extracted from minced tuna liver. Therefore, it was feasible to extract oil from high-moisture tuna liver by SDME.

Besides, the residual of DE was determined by SPME coupled with GC-MS, and the total ion chromatograms of DE and SDMEoil were shown in Fig. 3. The retention time of DE was $1.37 \mathrm{~min}$. However, DE was not detected in extracted liver oil. It was indicated that almost all the DE was removed from extracted liver oil.

\subsection{The effect of stirring}

Among three independent variables studied in our paper, the stirring speed greatly affected the yield of tuna liver oil, even the yield was mere $3.54 \pm 0.21 \%$ without stirring. Therefore, the effect of stirring was studied and analyzed specially. It was important to point out that the stirring rake was small and located above the minced fish liver (non-contacted, about $5 \mathrm{~cm}$ of distance). Thus the function of stirring was making subcritical DE rotational but not mixing subcritical DE and fish liver together.

Fig. 4(A) and (B) were the effect of stirring speed on the yield of liver oil and the picture of the residue in extraction tank, respectively. Under the conditions of 0 and $250 \mathrm{rpm}$, the yield was less than $5 \%$ and climbed no more after $40 \mathrm{~min}$, far less than the yield $(14.04 \pm 0.42 \%$ at $60 \mathrm{~min})$ under $500 \mathrm{rpm}$. Besides, complete differences were also observed between the residues with different stirring speeds. As shown in Fig. 4(B), the minced fish liver extracted under 0 and $250 \mathrm{rpm}$ turned into chunks, while it turned to powder under $500 \mathrm{rpm}$. Thus, under the conditions of 0 and $250 \mathrm{rpm}$, the low yield was because only the surface of tuna liver was extracted, and the high yield under $500 \mathrm{rpm}$ was because all the tuna liver was extracted. Considering that the tuna liver was high moisture $(61.41 \pm 3.12 \%)$ and extracted in a form of gloppy, it was hard for subcritical DE to penetrate into the interior of tuna liver and extract the lipids inside without stirring, which could be verified by the results above. The results indicated that stirring could enhance the penetration of subcritical DE, and the tuna liver could be extracted completely only when the stirring speed was fast 


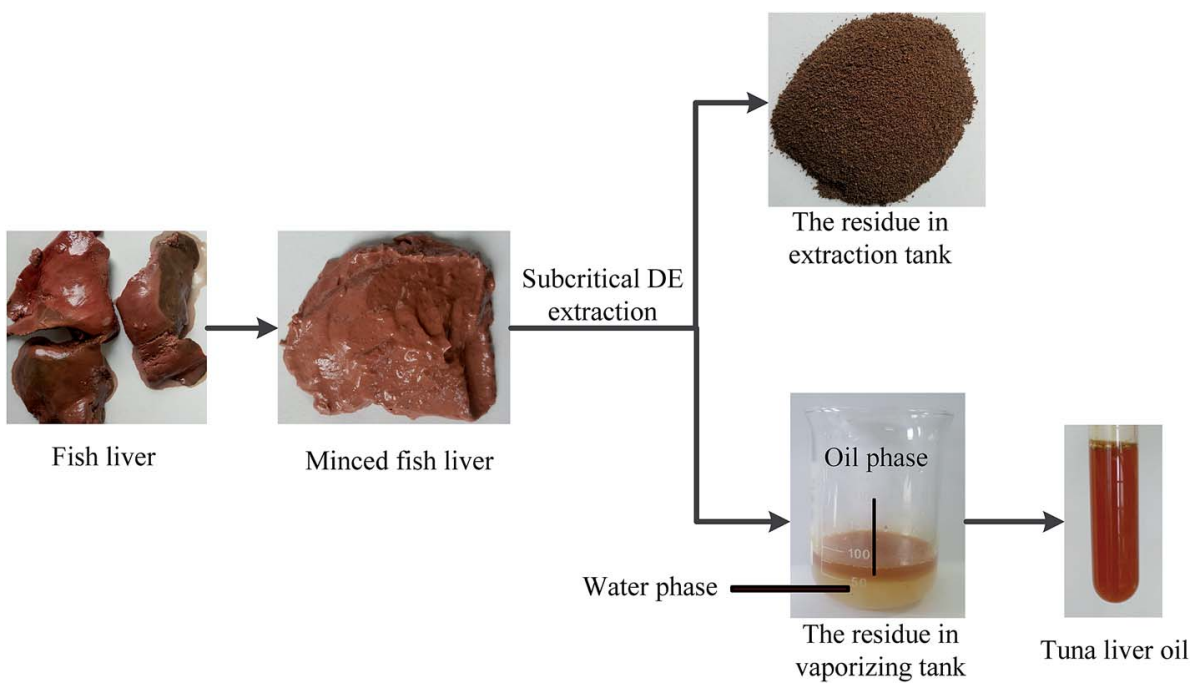

Fig. 2 The pictures of raw material and products at all stages of subcritical DE extraction.

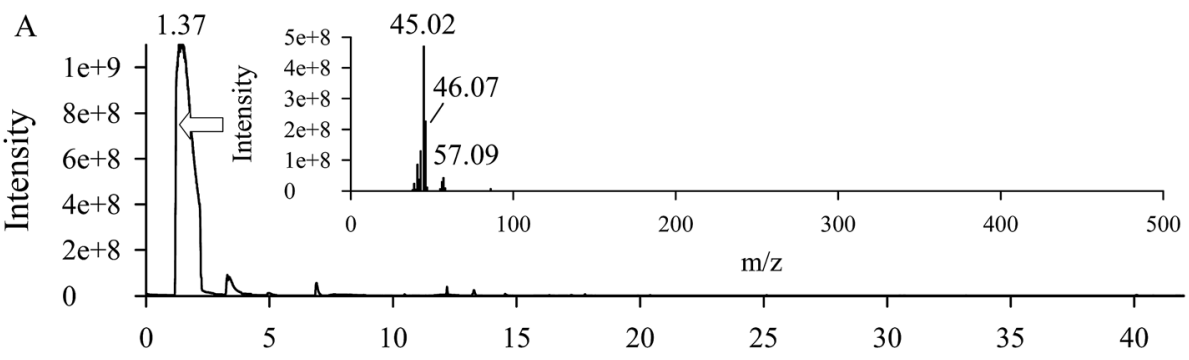

Time (min)

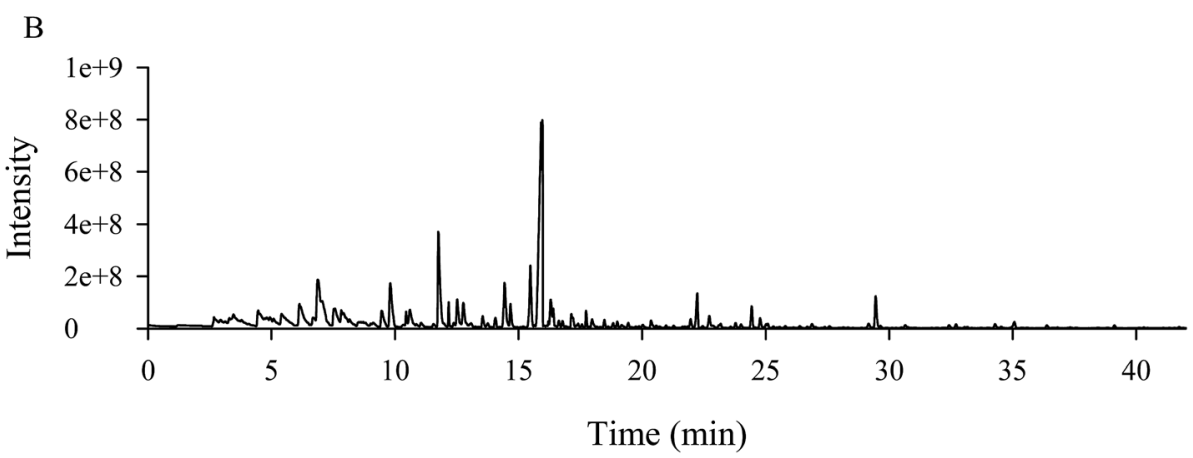

Fig. 3 The chromatograms of volatile compounds. (A) Dimethyl ether and the mass chromatogram at 1.37 min; (B) the tuna oil extracted by subcritical DE.

enough. Accordingly, the stirring speed in our study should be not less than $500 \mathrm{rpm}$, otherwise it may lead to the incomplete extraction.

\subsection{Analysis of the model}

The results obtained under different experiment conditions according to RSM design was listed in Table 1, which showed that the yield differed greatly depending on extraction conditions.
The multiple regression coefficients, including intercept, linear, quadratic, and interaction, were calculated by the least square technique, were summarized in Table 2. It was obvious that the linear and two quadratic parameters of the studied independent variables (temperature/pressure, time and stirring speed) were highly significant $(p<0.001)$. The interactions between temperature/pressure and time, temperature/pressure and stirring speed were not significant to the yield $(p>0.05)$. However, there was a significant interaction $(p<0.05)$ between time and stirring speed. Therefore, these results indicated that 

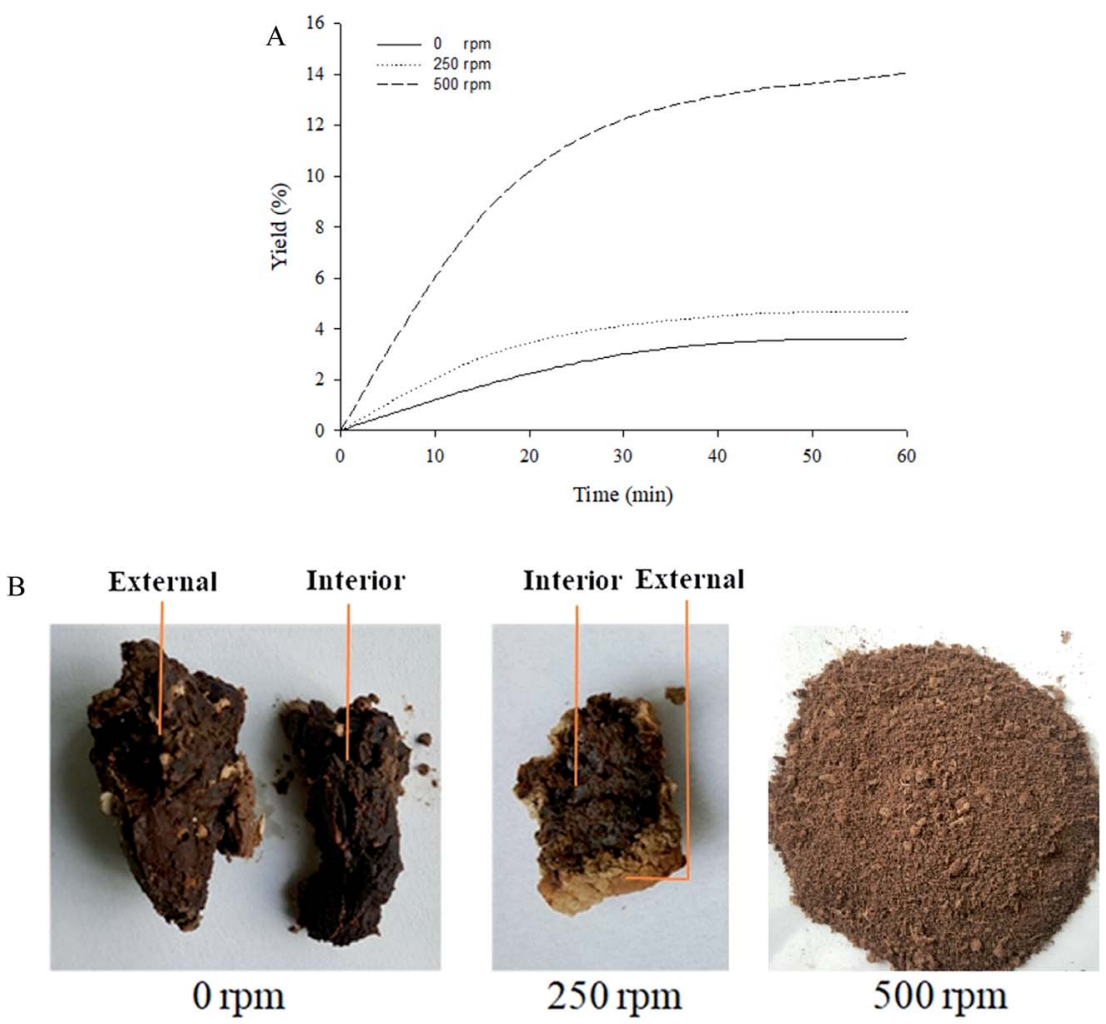

Fig. 4 The effect of stirring speed under $40{ }^{\circ} \mathrm{C}$. (A) The effect of stirring speed on the yield of liver oil, (B) part of the residue in extraction tank after 60 min extraction.

the oil yield was primary determined by the linear and quadratic effects of the studied independent variables.

The experimental data was analyzed by multiple regression, and the predicted yield of tuna liver oil $(Y)$ could be calculated according the below second-order polynomial model: $Y=16.85$ $+0.26 X_{1}+0.78 X_{2}+1.65 X_{3}-0.21 X_{2} X_{3}-0.43 X_{1}^{2}-0.90 X_{2}^{2}-$ $1.10 X_{3}^{2}$, where $X_{1}, X_{2}$, and $X_{3}$ are means of the coded factors of the temperature/pressure, time and stirring speed, respectively.

The analysis of variance and error for the response surface model were also listed in Table 2 . The determination coefficients $\left(R^{2}\right)$ and the correlation coefficients $(R)$ could reflect the quality of the model. For $R$, the closer to 1 , the better in the correlation between experimental and predicted values. ${ }^{23}$ The $R^{2}$ of our model was 0.9969 , which meant the good correlation between the model and experimental results. The error analysis indicated that the lack of fit was insignificant $(p>0.05)$. In other words, this model was adequate to predict the yield with different conditions of the variables. The coefficient of variation (CV\%) was 0.82 (below 5), indicating that the model was reproducible. ${ }^{19}$ The model's PRESS (predicted residual sum of squares), which was used to measure the matching of each point in the design with the model, was 1.49. The value of adeq. precision, indicated the signal to noise ratio of the model, was 49.430. Besides, the $F$-value of the model was high (251.40) and the $P$-value was extremely low $(<0.001)$. In summary, these results demonstrated that the model was credible and matched the experimental results well, which could be used for the following analysis.

\subsection{Effects and optimization of extraction conditions on extraction yield}

The three-dimensional response surfaces and two-dimensional contour plots for variables (temperature/pressure, time and stirring speed) to the oil yield were obtained according to the above model and shown in Fig. 5, which indicated the effects of extraction conditions on the yield of tuna liver oil.

Fig. 5(A) and (B) were the response surface and contour plots, respectively, indicated the effects of temperature/pressure and time on the oil yield with the stirring speed kept constant at $750 \mathrm{rpm}$. According to the results, time had a strong effect on the oil yield as compared with temperature/pressure. Time had a positive effect on the oil yield for the most of time, however, the negative effect revealed when the time was over $50 \mathrm{~min}$. Further time led to little change on the oil yield. As described in 2.3 , temperature had a positive linear effect on pressure, thus the pressure increased with the increase of temperature. Similar to $\mathrm{SC}-\mathrm{CO}_{2}$, the increases of temperature resulted in the reduction of the density of solvent, which led to the decrease of yield. However, the mass transfer speed increased with the increase of temperature, which could increase the yield. ${ }^{24}$ On the other hand, the oil solubility could improve by the increase of pressure due to the increased solvent density. ${ }^{25}$ Under these actions, the effect of the increased temperature/pressure on the oil yield was positive first and then turned to negative.

Fig. 5(C) and (D) were the response surface and contour plots, respectively, indicating the effect of temperature/pressure and stirring speed on the oil yield at the fixed time of $45 \mathrm{~min}$. 
Table 2 Estimated regression coefficients for the quadratic polynomial model and the analysis of variance (ANOVA) for the experimental results ${ }^{a}$

\begin{tabular}{|c|c|c|c|c|c|c|}
\hline Model & & & 37.53 & 9 & 4.17 & $251.40^{* * *}$ \\
\hline Intercept & 16.85 & 0.058 & & 1 & & \\
\hline$X_{2}$ & 0.78 & 0.046 & 4.82 & 1 & 4.82 & $290.63 * * *$ \\
\hline$X_{3}$ & 1.65 & 0.046 & 21.65 & 1 & 21.65 & $1305.18^{* * *}$ \\
\hline$X_{1} X_{2}$ & $2.500 \times 10^{-3}$ & 0.064 & $2.500 \times 10^{-5}$ & 1 & $2.500 \times 10^{-5}$ & $1.507 \times 10^{-3 \mathrm{NS}}$ \\
\hline$X_{1}^{2}$ & -0.43 & 0.063 & 0.78 & 1 & 0.78 & $46.88 * * *$ \\
\hline$X_{2}^{2}$ & -0.90 & 0.063 & 3.45 & 1 & 3.45 & $207.80 * * *$ \\
\hline$X_{3}^{2}$ & -1.10 & 0.063 & 5.12 & 1 & 5.12 & $308.42 * * *$ \\
\hline Residual & & & 0.12 & 7 & 0.017 & \\
\hline Lack of fit & & & 0.091 & 3 & 0.030 & $4.89^{\mathrm{NS}}$ \\
\hline Pure error & & & 0.025 & 4 & $6.320 \times 10^{-3}$ & \\
\hline
\end{tabular}

The effect of temperature/pressure on the oil yield was described above. As analyzed in 3.3, the increase of stirring speed could enhance the penetration of subcritical DE, and the tuna liver may extract incompletely when the stirring speed less than $500 \mathrm{rpm}$. For the larger stirring speed (in the range of 500$1000 \mathrm{rpm}$ ), the yield increased with the rise of stirring speed. This might be because the larger stirring speed could accelerate the speed of subcritical DE to penetrate into the tuna liver.

Fig. 5(E) and (F) were the response surface and contour plots, respectively, indicated the effect of time and stirring speed on the oil yield at the fixed temperature/pressure of $40 / 0.77^{\circ} \mathrm{C} / \mathrm{MPa}$. The effect of them on the oil yield both was described above. Besides, Fig. 5(F) indicated that extraction time was long when the oil yield reached maximum value at low stirring speed levels, however, the time was shorter at high stirring speed levels.

The conditions of SDME would be considered optimum when the oil yield reached maximum, which could be calculated according to the contour plots for the oil yield (Fig. 5). The optimum values of the test variables were as follows: temperature/pressure, $41.99 / 0.80{ }^{\circ} \mathrm{C} / \mathrm{MPa}$; time, $50.22 \mathrm{~min}$; stirring speed, $925.11 \mathrm{rpm}$. An oil yield of $17.59 \%$ was obtained under these conditions.

The trial experiments were carried out under optimized conditions. Taking convenience into account, the optimum test variables were changed slightly as follows: temperature/pressure, 42/0.80 ${ }^{\circ} \mathrm{C} / \mathrm{MPa}$; time, $50 \mathrm{~min}$; and stirring speed, $925 \mathrm{rpm}$. Besides, the predicted oil yield was $17.59 \%$ under the new conditions. The observed value was $17.46 \pm 0.23 \%$, which was reasonable as compared with the predicted value. These results indicated that the predicted value matched the experimental results well, which could also prove the validity of this model.

\subsection{Properties of the tuna liver oil}

3.6.1. Chemical properties. The chemical properties of the tuna liver oil extracted by SDME were shown in Table 3, as compared with the oil extracted by $\mathrm{SC}-\mathrm{CO}_{2}$.
SC- $\mathrm{CO}_{2}$ had been proved to be an advanced technology for extraction of high quality oil, which could reduce the hydrolysis of triacylglycerides and the oxidation of fatty acids. ${ }^{26}$ Therefore, the oil obtained by $\mathrm{SC}-\mathrm{CO}_{2}$ presented lower acid value and peroxide value, as compared with tradition extraction methods (cold extraction, wet extraction and enzymatic extraction). ${ }^{4}$ However, no significant differences $(p<0.05)$ were observed in acid values and peroxide values between SDME and $\mathrm{SC}-\mathrm{CO}_{2}$ oil. The non-saponification matter means the content of nonglyceride-soluble matter. The non-saponification matter was significantly higher $(p<0.05)$ in oil extracted with SDME than SC- $\mathrm{CO}_{2}$, which indicated that more non-glyceride-soluble matter was extracted by SDME. Iodine value reflected the unsaturation of fish oil, and no significant differences $(p<0.05)$ were observed between the oils obtained by these two extraction methods, the same as insoluble impurities. However, the moisture and volatile matter were significantly higher $(p<0.05)$ in oil extracted with SDME than with $\mathrm{SC}^{-\mathrm{CO}_{2}}$. Different from supercritical $\mathrm{CO}_{2}$, the water and lipids were co-extracted by subcritical DE, and could be easily separated by centrifugation, thus there were traces of water residue.

Above all, the quality of subcritical DE-oil was similar with supercritical $\mathrm{CO}_{2}$-oil, except non-saponification matter, and moisture and volatile matter were a little bit higher. The results indicated that the high quality oil could also be produced by SDME.

3.6.2. Fatty acid composition. Besides, fatty acid composition analysis was carried out. The total ion chromatogram of tuna liver oil fatty acids was shown in Fig. S5 $\dagger$ and Table 4 shows the composition of fatty acids in the tuna liver oil with different extraction methods.

According to the report of Hao et al., ${ }^{27} \mathrm{SC}^{-\mathrm{CO}_{2}}$ was also shown the advantage in fatty acid composition when compared with wet extraction and enzymatic extraction. For the oil obtained by $\mathrm{SC}-\mathrm{CO}_{2}$, the higher ratios of PUFA and monounsaturated fatty acid (MUFA) were observed. While in fatty acid 
A

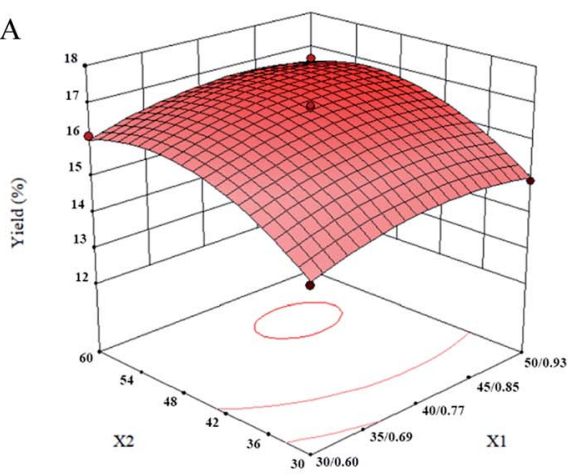

C

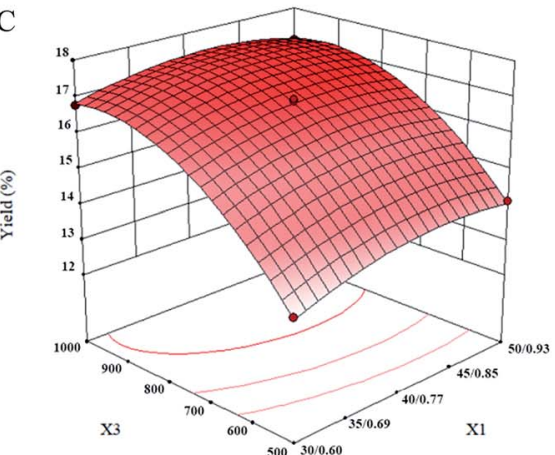

$\mathrm{E}$

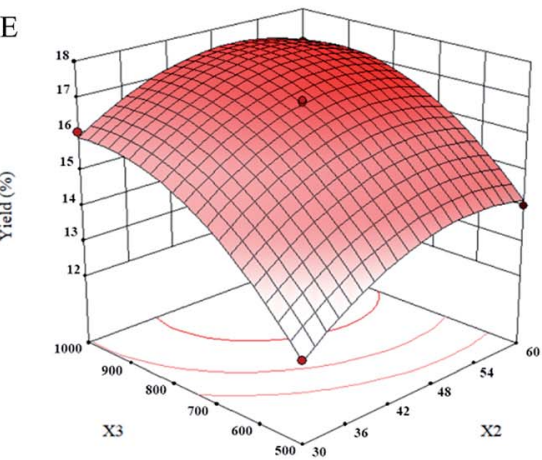

B

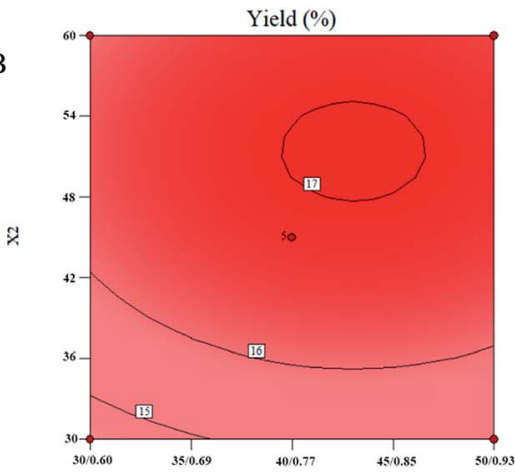

$\mathrm{x} 1$

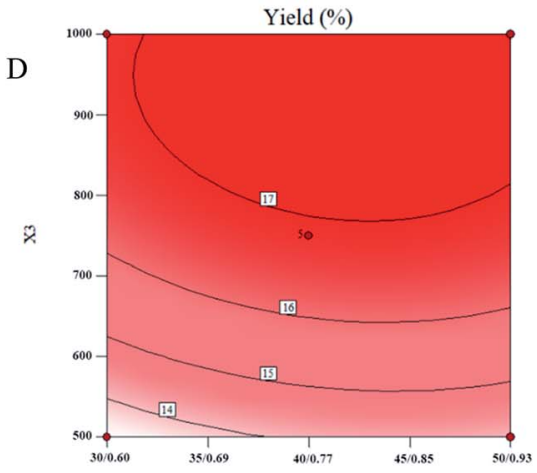

$\mathrm{x}_{1}$

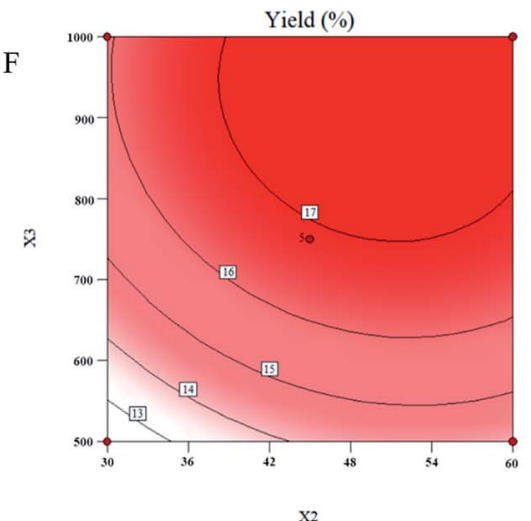

Fig. 5 Response surface plots $(A, C$ and $E)$ and contour plots $(B, D$ and $F)$ of the oil yield affected by temperature/pressure $\left(X_{1}\right)$, time $\left(X_{2}\right)$ and stirring speed $\left(X_{3}\right)$.

Table 3 Chemical properties of tuna liver oil with different extraction methods

\begin{tabular}{lcc}
\hline Property & Subcritical DE & Supercritical $\mathrm{CO}_{2}$ \\
\hline Acid value $\left(\mathrm{mg} \mathrm{KOH} \mathrm{g}{ }^{-1}\right)$ & $1.87 \pm 0.26^{a}$ & $1.51 \pm 0.13^{a}$ \\
Peroxide value $\left(\mathrm{mmol} \mathrm{kg}^{-1}\right)$ & $2.18 \pm 0.11^{a}$ & $2.21 \pm 0.20^{a}$ \\
Non-saponification matter $(\%)$ & $2.01 \pm 0.13^{a}$ & $1.16 \pm 0.08^{b}$ \\
Moisture and volatile matter $(\%)$ & $0.86 \pm 0.08^{a}$ & $0.15 \pm 0.03^{b}$ \\
Iodine value (g/100 g) & $147.2 \pm 2.8^{a}$ & $148.4 \pm 1.7^{a}$ \\
Insoluble impurities (\%) & $1.22 \pm 0.09^{a}$ & $1.11 \pm 0.13^{a}$
\end{tabular}

${ }^{a}$ Means followed by different letters in the same row demonstrated significant difference $(p<0.05)$. Each value is the mean \pm standard deviation of triplicate determinations. ${ }^{b}$ Means followed by different letters in the same row demonstrated significant difference $(p<0.05)$. Each value is the mean \pm standard deviation of triplicate determinations. composition, no significant differences were observed for oils extracted by SDME and SC-CO .

To the oil obtained by SDME, the total saturated fatty acid (SFA) content was $41.03 \pm 0.78 \%$, and the content of palmitic acid $(27.79 \pm 0.74 \%)$ was the highest among them. However, the content of total MUFA was $23.82 \pm 0.17 \%$. The most prevalent MUFA was oleic acid $(16.45 \pm 0.37 \%)$. To PUFA, the total content was $30.29 \pm 0.49 \%$, and the contents of DHA and EPA were $16.52 \pm 0.45 \%$ and $4.02 \pm 0.14 \%$, respectively. A value of $4.86 \pm 0.18 \%$ represented the percentage of the unknown fatty acids in the tuna liver oil. Similar to the results of Kang et al., ${ }^{22}$ palmitic acid, oleic acid and DHA were primary fatty acids in tuna liver oil, followed by stearic acid, palmitoleic acid and EPA. 
Table 4 Composition of fatty acids (area\%) of tuna liver oil with different extraction methods

\begin{tabular}{|c|c|c|}
\hline Fatty acid & Subcritical DE & Supercritical $\mathrm{CO}_{2}$ \\
\hline C14:0 & $1.29 \pm 0.09$ & $1.41 \pm 0.10$ \\
\hline C15:0 & $1.03 \pm 0.11$ & $1.00 \pm 0.01$ \\
\hline C16:0 & $27.79 \pm 0.74$ & $28.45 \pm 0.64$ \\
\hline $\mathrm{C} 17: 0$ & $2.33 \pm 0.17$ & $2.23 \pm 0.01$ \\
\hline C18:0 & $8.34 \pm 0.10$ & $8.56 \pm 0.66$ \\
\hline C20:0 & $0.25 \pm 0.02$ & $0.25 \pm 0.03$ \\
\hline C16:1 (n-7) & $5.56 \pm 0.24$ & $5.26 \pm 0.14$ \\
\hline C16:1 (n-9) & $0.30 \pm 0.02$ & $0.29 \pm 0.02$ \\
\hline C18:1 (n-9) & $16.45 \pm 0.37$ & $16.09 \pm 0.74$ \\
\hline C18:1 (n-7) & $1.11 \pm 0.09$ & $0.96 \pm 0.05$ \\
\hline $\mathrm{C} 20: 1(\mathrm{n}-9)$ & $0.39 \pm 0.07$ & $0.34 \pm 0.05$ \\
\hline C18:2 (n-6) & $0.94 \pm 0.15$ & $0.92 \pm 0.01$ \\
\hline C18:3 (n-3) & $1.93 \pm 0.10$ & $2.15 \pm 0.10$ \\
\hline C18:4 (n-3) & $0.71 \pm 0.04$ & $0.69 \pm 0.02$ \\
\hline $\mathrm{C} 20: 2(\mathrm{n}-6)$ & $0.26 \pm 0.04$ & $0.20 \pm 0.02$ \\
\hline $\mathrm{C} 20: 3(\mathrm{n}-3)$ & $0.54 \pm 0.08$ & $0.62 \pm 0.01$ \\
\hline $\mathrm{C} 20: 4(\mathrm{n}-6)$ & $3.24 \pm 0.15$ & $3.54 \pm 0.07$ \\
\hline $\mathrm{C} 20: 4(\mathrm{n}-3)$ & $0.16 \pm 0.02$ & $0.21 \pm 0.02$ \\
\hline $\mathrm{C} 20: 5$ (n-3, EPA) & $4.02 \pm 0.14$ & $3.88 \pm 0.09$ \\
\hline $\mathrm{C} 21: 5(\mathrm{n}-6)$ & $0.68 \pm 0.05$ & $0.63 \pm 0.01$ \\
\hline $\mathrm{C} 22: 5(\mathrm{n}-6)$ & $0.71 \pm 0.02$ & $0.86 \pm 0.04$ \\
\hline C22:5 (n-3) & $0.58 \pm 0.03$ & $0.50 \pm 0.02$ \\
\hline C22:6 (n-3, DHA) & $16.52 \pm 0.45$ & $16.06 \pm 0.38$ \\
\hline$\sum$ SFA & $41.03 \pm 0.78^{a}$ & $41.89 \pm \mathbf{0 . 0 6}^{a}$ \\
\hline$\sum$ MUFA & $23.82 \pm 0.17^{a}$ & $22.94 \pm 0.69^{a}$ \\
\hline$\sum$ PUFA & $30.29 \pm 0.49^{a}$ & $30.28 \pm 0.22^{a}$ \\
\hline n-3/n-6 ratio & $4.20 \pm 0.24^{a}$ & $3.92 \pm 0.08^{a}$ \\
\hline Unknown & $4.86 \pm 0.18$ & $4.89 \pm 0.44$ \\
\hline
\end{tabular}

Thus, the obtained tuna liver oil by SDME, contained considerable amounts of oleic acid, DHA and EPA, was valuable and could increase the added value of the product.

\section{Conclusions}

The results indicated that it was feasible to extract oil from highmoisture tuna liver by SDME. Besides, the second-order polynomial model was credible, which could describe and predict the change of tuna liver oil yield with different conditions in the experimental ranges. In addition, stirring speed strongly affected the yield of oil. Because stirring could enhance the penetration of subcritical DE, and the tuna liver could be extracted completely only when the stirring speed was fast enough. The optimum test variables were as follows: temperature/pressure, $42 / 0.80{ }^{\circ} \mathrm{C} / \mathrm{MPa}$; time, $50 \mathrm{~min}$; stirring speed, $925 \mathrm{rpm}$. Under these conditions, the observed oil yield was $17.46 \pm 0.23 \%$. Except non-saponification matter and moisture and volatile matter, no significant differences were observed between the oils obtained by SDME and $\mathrm{SC}-\mathrm{CO}_{2}$ within determined indexes. The results indicated the quality of subcritical DE-oil was similar with supercritical $\mathrm{CO}_{2}$-oil. However, the pressure employed in SDME was 0.80 MPa, far less than which employed in $\mathrm{SC}-\mathrm{CO}_{2}(35 \mathrm{MPa})$. Besides, time- and energy-consuming freeze-drying, which was necessary before SC- $\mathrm{CO}_{2}$, was removed in SDME. SDME proved to be a promising technique to extract oil from the tuna liver.

\section{Conflicts of interest}

The authors declare that they have no competing interests.

\section{Acknowledgements}

This work was financially supported by the National Natural Science Foundation of China (No. 31671880) and Primary Research and Developement Plan of Zhejiang Province (No. 2015C02033).

\section{References}

1 J. Choi, Proc. Nutr. Soc., 2013, 72, 265.

2 G. Navarro-García, R. Pacheco-Aguilar, L. Bringas-Alvarado and J. Ortega-García, Food Chem., 2004, 87, 89-96.

3 M. J. Dibley and D. A. Jeacocke, Food Nutr. Bull. Suppl., 2017, 22, 267-284.

4 N. Rubiorodríguez, S. M. de Diego, S. Beltrán, I. Jaime, M. T. Sanz and J. Rovira, J. Food Eng., 2012, 109, 238-248.

5 N. Rubio-Rodríguez, S. M. D. Diego, S. Beltrán, I. Jaime, M. T. Sanz and J. Rovira, J. Supercrit. Fluids, 2008, 47, 215226.

6 M. Goto, H. Kanda, W. Wahyudiono and S. Machmudah, J. Supercrit. Fluids, 2015, 96, 245-251.

7 X. D. Fan, Y. Hou, X. X. Huang, T. Q. Qiu and J. G. Jiang, J. Agric. Food Chem., 2015, 63, 4597.

8 A. B. Zanqui, D. R. de Morais, S. C. Da, J. M. Santos, S. T. Gomes, J. V. Visentainer, M. N. Eberlin, L. CardozoFilho and M. Matsushita, Food Chem., 2015, 188, 452-458.

9 E. F. S. Authority, EFSA J., 2009, 7, 3-13.

10 B. Nagy and B. Simándi, J. Supercrit. Fluids, 2008, 46, 293298.

11 L. Peng, H. Kanda and H. Makino, Fuel, 2014, 116, 370-376.

12 H. Kanda and H. Makino, J. Environ. Sci. Eng., 2009, 4, 356361.

13 O. Catchpole, J. Ryan, Z. Yin, K. Fenton, J. Grey, M. Vyssotski, A. Mackenzie, E. Nekrasov and K. Mitchell, J. Supercrit. Fluids, 2010, 53, 34-41.

14 H. Kanda, H. Makino, M. Morita, K. Takegami, N. Takeda and K. Oshita, J. Jpn. Soc. Mater. Cycles Waste Manage., 2008, 19, 409-413.

15 H. Kanda, P. Li and H. Makino, Food Bioprod. Process., 2013, 91, 376-380.

16 H. Kanda, Y. Kamo, S. Machmudah, E. Y. Wahyudiono and M. Goto, Mar. Drugs, 2014, 12, 2383.

17 F. Sahena, I. S. M. Zaidul, S. Jinap, A. M. Yazid, A. Khatib and N. A. N. Norulaini, Food Chem., 2010, 120, 879-885.

18 J. B. Jentzer, M. Alignan, C. Vacagarcia, L. Rigal and G. Vilarem, Food Chem., 2015, 166, 561.

19 Z. Liu, L. Mei, Q. Wang, Y. Shao and Y. Tao, LWT-Food Sci. Technol., 2014, 56, 168-174.

20 AOCS, American Oil Chemists' Society, 2009, pp. 32-286. 
21 Y. Aquerreta, I. Astiasarán, A. Mohino and J. Bello, Food Chem., 2002, 77, 147-153.

22 K. T. Kang, S. H. Min, S. J. Jee, J. H. Lee, H. S. Kim and J. S. Kim, Food Sci. Biotechnol., 2007, 16, 367-373.

23 V. Pujari and T. S. Chandra, Process Biochem., 2000, 36, 31-37.

24 S. Liu, Y. Feng, C. Zhang, H. Ji, P. Hong and C. Deng, J. Supercrit. Fluids, 2009, 48, 9-14.
25 W. Y. Lee, Y. J. Cho, L. O. Sang, J. H. Park, W. S. Cha, J. Y. Jung and H. C. Yong, Food Sci. Biotechnol., 2000, 9, 174-178.

26 M. Létisse, M. Rozières, A. Hiol, M. Sergent and L. Comeau, J. Supercrit. Fluids, 2006, 38, 27-36.

27 S. Hao, Y. Wei, L. Li, X. Yang, J. Cen, H. Huang, W. Lin and X. Yuan, Food Chem., 2015, 173, 274-282. 